\title{
A crise de meia idade no homem: Aspectos fisiológicos e psicológicos
}

\author{
The crisis of middle age in men: Physiological and psychological aspects \\ La crisis de la mediana edad en los hombres: Aspectos fisiológicos y psicológicos
}

Recebido: 20/04/2021 | Revisado: 26/04/2021 | Aceito: 28/04/2021 | Publicado: 13/05/2021

Thalyta Freitas dos Santos Laguna

ORCID: https://orcid.org/0000-0002-4227-0020

Universidade Franciscana, Brasil

E-mail: thalytalaguna@gmail.com

Telma Garcez Leal

ORCID: https://orcid.org/0000-0003-3279-3044 Universidade Franciscana, Brasil

E-mail: telmagarcezleal@hotmail.com

Aline Priscila Subutzki Lemos

ORCID: https://orcid.org/0000-0002-0472-4474

Universidade Franciscana, Brasil

E-mail: asubutzki@hotmail.com

Caroline Joana Gerhardt

ORCID: https://orcid.org/0000-0001-9453-2371

Universidade Franciscana, Brasil

E-mail: caroljgerhardt@gmail.com

Bruna Souza Gelain Cocco

ORCID: https://orcid.org/0000-0002-9387-5177

Universidade Franciscana, Brasil

E-mail: bruna.gcocco@gmail.com

Luciane Najar Smeha

ORCID: https://orcid.org/0000-0002-3068-3776

Universidade Franciscana, Brasil

E-mail: lucianenajar@yahoo.com.br

\begin{abstract}
Resumo
A meia idade, etapa da vida na qual ocorrem transformações que direcionam o ser humano para a chamada madurescência, ocorre entre 40 e 60 anos. Durante este período, uma série de transformações tão importantes quanto às que ocorrem na adolescência, marca o indivíduo que vivencia essa fase. Outrossim, vale salientar que estas mudanças se apresentam com caraterísticas distintas nos homens e nas mulheres. Este artigo tem por objetivo discorrer sobre a crise de meia idade no homem, analisando, para tanto, os aspectos físicos característicos da fase, bem como os psicológicos. Além disso, abordar-se-á a relação com o trabalho e aposentadoria. À luz da literatura, apresentar-se-á como esta fase de intensos questionamentos e reflexões se manifesta no homem. Trata-se de um estudo de revisão narrativa da literatura, para o qual, artigos, livros e dados estatísticos foram consultados a fim de se elucidar e trazer reflexões críticas acerca do conteúdo abordado. Observou-se como principais resultados, que a madurescência é uma fase de intensos questionamentos e reflexões, circundada, sobretudo, pelas inúmeras transformações no corpo e no psicológico. Entretanto, embora seja um tema que abre margem para diferentes abordagens de pesquisa, poucos são os estudos atuais dedicados especificamente à crise de meia-idade no sexo masculino. Destarte, conclui-se, ao final do estudo, que com a ampliação da expectativa de vida, este período tornouse ainda mais valioso e primordial à qualidade de vida, saúde, prazer e autoestima que influenciarão, sobremaneira, na velhice.
\end{abstract}

Palavras-chave: Fase; Homem; Madurescência; Meia-idade; Masculino.

\begin{abstract}
Middle age, the stage of life in which there are transformations that direct the human being towards the old age, takes place, according to the literature, between 40 and 60 years old. During this period, a series of transformations, as important as those that occur in adolescence, marks the individual who experiences this phase. Likewise, it is worth noting that these changes present different characteristics in men and women. That said, this article aims to discuss the crisis of middle age in men, paying attention to the changes that have occurred in terms of physical and psychological aspects, and not least, how they relate to work and retirement. This study consists in a narrative review of the literature, elaborated in a period of 2 months, during which, articles, books and statistical data were consulted in order to elucidate and bring critical reflections on the content covered. It was observed, as main results, that the maturity is a phase of intense questions and reflections, surrounded, above all, by countless transformations in the body, mind and
\end{abstract}


individual psychology. However, although it is a topic that opens the door to different research approaches, there are few current studies dedicated specifically to the midlife crisis in males. Thus, we concluded, at the end of the study, that with the expansion of life expectancy, this period became even more valuable and essential to the quality of life, health, pleasure and self-esteem, that will greatly influence the old age.

Keywords: Phase; Man; Madurescence; Middle-age; Male.

\section{Resumen}

La mediana edad, etapa de la vida en la cuál ocurren transformaciones que direccionan el ser humano para la llamada madurescencia, ocurre, según la literatura, entre 40 y 60 años. Durante este periodo, una serie de transformaciones tan importantes cuanto las que ocurren en la adolescencia, marca el individuo que vivencia esta fase. Además vale resaltar que estos cambios se presentan con características distintas en los hombres y las mujeres. Dicho esto, este articulo tiene como objetivo discutir sobre la crisis de mediana edad en el hombre, estando atentos a las transformaciones ocurridas, prestando atención a los aspectos físicos y psicológicos, y no menos importante, como ocurre su relación con el trabajo y la jubilación. Se trata de un estudio de revisión narrativa de literatura, elaborado en un periodo de 2 meses, durante los cuales, artículos, libros y datos estatísticos fueron consultados para dilucidar y aportar reflexiones críticas sobre el contenido cubierto. Se observaron como principales resultados, que la madurescencia es una fase de intensos cuestionamientos y reflexiones, rodeada, sobretodo, por las innumerables transformaciones en el cuerpo, mente y psicológico. Sin embargo, aunque sea un tema que abre margen para diferentes enfoques de investigación, son pocos los estúdios actuales dedicados específicamente a la crisis de la mediana edad masculina. Por lo tanto, concluimos, al final del estudio, que con la expansión de la expectativa de vida, este periodo se volvió aún más valioso y primordial a la calidad de vida, salud, placer y autoestima que van a influenciar de gran manera en la vejez.

Palabras clave: Fase; Hombre; Madurescencia; Edad Media; Masculino.

\section{Introdução}

A madurescência ou meia-idade, como é conhecida popularmente, diz respeito ao período de vida do ser humano que se encontra entre o final da vida adulta e o início da terceira idade, englobando a faixa etária de 40 a 60 anos em média (Eizirik \& Bassols, 2013). Segundo os autores, a madurescência é o processo de transformação rumo a maturidade. Esse momento é tão importante para o desenvolvimento do indivíduo, quanto a fase da adolescência, pois em ambas as ocasiões acontecem significativas mudanças biológicas e psicológicas. Ainda, conforme esses autores, essas duas etapas da vida não se caracterizam apenas como o adiamento da procriação (adolescência) e da morte (madurescência), mas dizem respeito também ao crescimento e desenvolvimento psíquico (Eizirik \& Bassols, 2013).

Estudos acerca da meia idade demonstram que as mudanças dessa fase ocorrem de formas diferentes entre os gêneros feminino e masculino, evidenciando que cada qual enfrentará essa fase a partir das peculiaridades próprias de seus organismos, bem como por fatores sociais (Gonçalves, Fagulha \& Ferreira, 2005; Sartori \& Zilberman, 2009; Virgolino et al., 2013). Silva e Silva (2014) revelam a importância de estudar a madurescência masculina, visando compreender as transformações intrínsecas à idade, muito porque a sociedade está impregnada de pressões e julgamentos neste período da vida, os quais implicam especialmente na dificuldade dos homens para demostrarem a fragilidade inerente ao ser humano nas diferentes fases do amadurecimento. Desse modo, a madurescência masculina está atravessada pela manutenção do papel de provedores da família, enquanto vivenciam uma crise de meia-idade que pode se potencializar na velhice (Silva \& Silva, 2014; Trindade, 2002).

As mudanças relacionadas ao corpo físico são visualmente perceptíveis, como cabelos grisalhos, rugas, perdas de cabelo e tônus muscular, sendo definidas cronologicamente, como apontam Antunes e Silva (2013), ao mencionarem que nos descritores da saúde (DeCS) e também na Organização Mundial da Saúde (OMS) estas são características da meia-idade. Para mais, as mudanças físicas geram uma percepção consciente do próprio envelhecimento, enquanto as psicológicas estão direcionadas para o luto - perda de entes, perda de papéis sociais, perda do corpo, perda do trabalho - promovendo a mudança psíquica e a (re)significância do amadurecimento (Montero, 2015).

Isso posto, é na aposentadoria que se concentra a maior dificuldade masculina durante essa transição, já que por muito tempo foi impresso socialmente nos homens o atributo de provedores de família. Conforme Ferreira (2008), a atenção 
masculina na madurescência fica mais voltada para o trabalho, planos financeiros e aposentadoria. $\mathrm{O}$ contato com outras pessoas, as participações sociais e o sentimento de utilidade, além de benefícios econômicos, está mais facilmente assegurado no trabalho do que fora dele (Paiva, 2016). Sartori e Zilberman (2013) afirmam que os aspectos culturais relacionados à masculinidade produzem um enorme sofrimento aos homens, porém esse é silencioso, e, comumente gerador de casos de alcoolismo nessa fase da vida, hospitalizações psiquiátricas, suicídios, além de sérios problemas de saúde física (Sartori \& Zilberman, 2009). E entrando na linha do idoso, estes predicativos não servem mais. Muito pelo contrário, viram antônimos.

Destarte, independentemente desse momento trazer consigo um ciclo de encerramento, faz-se necessário compreender que na verdade trata-se de um novo começo, afinal o aumento da expectativa de vida atrelado a melhoria de qualidade desta, trouxe consigo novos horizontes para as pessoas que se encontram na madurescência. Como bem nos lembra Montero (2015), nesse período de vida, ao invés de se entregar à morte, o indivíduo opta por prolongar sua vida, tanto quanto possível. Para ele, na maturidade há uma chance, oferecida pelo ciclo de vida, para que o sujeito consiga promover, continuar e aprofundar o desenvolvimento individual na sua subjetividade, além dos vínculos com objetos e o intercâmbio de gerações.

Este estudo justifica-se, uma vez que é premente ampliar reflexões sobre as transformações vivenciadas na madurescência masculina nas esferas físicas, psicológicas e sociais, investigando-se a dinâmica relação entrelaçada ao campo cultural e, especialmente, pensar a respeito das demandas que levam um homem a sofrer na maioria das vezes silenciosamente.

A partir dessas considerações, este trabalho tem por objetivo discorrer sobre a crise de meia idade no homem, analisando, para tanto, os aspectos físicos característicos da fase, bem como os psicológicos. Além disso, abordar-se-á como se dá a relação com o trabalho e aposentadoria. À luz da literatura, apresentar-se-á como esta fase de intensos questionamentos e reflexões se manifesta no homem.

\section{Metodologia}

A fim de discorrer sobre a crise de meia idade no homem, optou-se pela revisão narrativa da literatura que possibilita, dentre outros fatores, a oportunidade de trabalhar temáticas abertas, uma vez que não exige um protocolo rígido e cujas fontes não são pré-determinadas (Cordeiro, Oliveira, Rentería e Guimarães, 2007). Estudos com este tipo de revisão são amplos e adequados para descrever o estado da arte de determinado assunto, a partir de um ponto de vista contextual ou teórico, onde é possível imprimir a crítica dos autores (Rother, 2007).

Constituída por uma análise ampla da literatura, a revisão narrativa é de fundamental importância, uma vez que através dela torna-se possível adquirir e atualizar conhecimentos acerca de determinadas temáticas, evidenciando novos métodos, ideias e subtemas, ampliando estudos e compondo abstrações (Elias et al., 2012).

Assim, para o desenvolvimento deste trabalho, utilizou-se da pesquisa bibliográfica realizada em livros digitais e impressos, estatísticas dos principais Institutos de Pesquisa oficiais e artigos científicos publicados em periódicos nacionais e internacionais. Tais publicações foram pesquisadas nas plataformas Scientific Eletronic Library Online (Scielo), Biblioteca Virtual em Saúde (BVS) e Literatura Latino Americana e do Caribe em Ciências da Saúde (LILACS) através dos descritores: meia idade, madurescência, masculina, homem, crise de meia idade. Além disso, foram consideradas monografias, dissertações, teses e boletins informativos. A pesquisa, de abordagem qualitativa, foi realizada no período de maio e junho de 2020.

\section{Resultados e Discussão}

\section{Aspectos Físicos}

Desde os primórdios, dentro de uma perspectiva heterossexuada do mundo, as relações homens/mulheres e mulheres/homens perpassam o conceito propagado pelo modelo naturalista de entendimento, no qual os homens possuem um 
caráter dominador, forte, viril, provedor e invulnerável. Neste sentido, dentro da historicidade da masculinidade, esses conceitos foram impressos na sociedade, convergindo assim para a ideia de que o homem possui uma abertura maior no meio em que vive, e que pode se expor - dado o seu caráter de invulnerabilidade - a muito mais riscos, se comparados às mulheres. Estas, em contrapartida, são talhadas mais para o cuidado com o outro e consigo mesmas, o que nos leva ao entendimento de que estas questões estão enraizadas na nossa cultura paternalista (Gomes, Nascimento \& Araújo, 2007).

Conforme apontamentos da Política Nacional de Atenção à Saúde Integral do Homem (Brasil, 2008), quase um terço dos homens brasileiros não vai ao médico regularmente, indicando, em boa ou em sua maior parte, que ainda é por uma questão cultural. Tal situação os faz padecer de condições severas e crônicas de saúde, levando-os muitas vezes a morte. Para Lemos et al. (2017), entende-se, dentre outros aspectos, que o imaginário social e o modo como o homem é visto, contribuem de maneira significativa para que ele pouco procure os serviços de saúde e se cuide menos. Gomes, Nascimento e Araújo (2007) destacam que essa "procura" pouco tem a ver com oferta e demanda de serviços, mas sim com os hábitos de prevenção, que está muito mais relacionado às mulheres do que aos homens.

Uma das principais consequências disso é que no Brasil, homens vivem, em média, sete anos menos que as mulheres, conforme aponta o Instituto Brasileiro de Geografia e Estatística (IBGE, 2019). Segundo consta na Política Nacional de Atenção à Saúde Integral do Homem (Brasil, 2008), se os homens realizassem exames, de maneira preventiva, bem como se apresentassem maior comprometimento com os tratamentos de doenças crônicas, muitos agravos poderiam ser evitados. Para a política, isso implica em mudança de comportamentos e hábitos de vida, fatores que não possuem tanta aderência entre os homens. Correa, Alcaças e Assis (2020), salientam que o estilo de vida, incluindo o uso excessivo de álcool, pode colocá-los em risco de incapacidades durante o curso da vida, com consequências severas principalmente em idade avançada.

Para Lemos et al. (2017) e Quadros e Schiavani (2005), ainda que portadores de variados tipos de enfermidades como diabetes, hipertensão, obesidade, doenças hepáticas provenientes ou não do abuso de álcool, dentre outras - também sejam negligentes com o autocuidado, vale salientar que há outra questão importante que reforça a baixa procura pelos serviços de saúde: o fato que as grandes variações hormonais em alguns homens deverão surgir somente na velhice. Desta forma, estes indivíduos não percebem a necessidade de realizar exames preventivos por não apresentarem sintomas, colocando assim a sua saúde em risco por não reconhecerem suas próprias necessidades (Lemos et al., 2017; Quadros e Schiavani 2005).

Segundo Filho et al. (2015), a menopausa, caracterizada pela interrupção definitiva do ciclo ovulatório, marca o fim do período fértil feminino. Entretanto, no homem, o fenômeno análogo - a interrupção definitiva da produção de espermatozoides - não existe, porém, a literatura aponta que pessoas do sexo masculino experimentam um declínio gradual de suas funções gônadais ao longo da vida, caracterizada pela diminuição dos níveis de testosterona sérica e pela pouca produção de espermatozoides (Bulcao et al., 2004).

Diante disso, diferentes categorias/terminologias diagnósticas são empregadas no meio médico para caracterizar o declínio hormonal masculino relacionado ao envelhecimento: andropausa, deficiência androgênica do envelhecimento masculino (ADAM ou DAEM) e, mais recentemente, síndrome da deficiência de testosterona (TDS ou SDT) (Thiago, Russo \& Camargo Júnior, 2016).

Para a literatura médica, o envelhecimento do homem é acompanhado de uma progressiva diminuição do número de seus andrógenos, dentre os quais destaca-se a testosterona, cuja deficiência pode afetar o corpo humano como um todo, e não apenas a sexualidade do indivíduo (Glina, Puech-Leão, Reis \& Pagani, 2002).

A ciência aponta e comprova que todas as células do organismo precisam desse poderoso hormônio e que sua escassez ou ausência, além de trazer severas consequências, é associado à quase todas as doenças degenerativas (Finkelstein et al., 2013). A partir da puberdade, ela desempenha um papel muito relevante, pois ativa a produção de sêmen, faz também com que o pênis cresça, os pelos do corpo aumentem, a voz engrosse, além de auxiliar no desenvolvimento da próstata, glândula de 
fundamental importância no sistema genital masculino (Glina, Puech-Leão, Reis \& Pagani, 2002). Ao longo dos anos, esse hormônio colabora também para a manutenção da massa muscular, do tecido ósseo, afetando também, o estado emocional. A progressiva diminuição das taxas de andrógenos - em especial de testosterona - implica no surgimento da andropausa (Quadros \& Schiavani, 2005).

O declínio da concentração sérica de testosterona inicia quando o homem está por volta dos 40 anos de idade, porém, a andropausa não é um fenômeno universal, ou seja, não necessariamente acomete todos os homens. Aproximadamente 10\% dos homens entre 40 e 50 anos apresentam níveis subnormais de testosterona, cifra que ascende a 30\% em homens mais maduros; já com a idade na faixa dos 60-69 a porcentagem dos homens com andropausa sobe para 40\% (Bonaccorsi, 2001).

Apesar de um decréscimo na concentração sérica de testosterona ser um fenômeno que atinge praticamente a totalidade dos homens, nem todos apresentam sintomas relacionados. Contudo, naqueles que apresentam indícios, diversas são as manifestações que podem vir a ocorrer. Entre elas podemos explicitar, conforme apontam Quadros e Schiavani (2005) na tabela 1.

Tabela 1 - Manifestações decorrentes da baixa na concentração sérica de testosterona.

ESFERA SEXUAL

ESFERA FÍSICA
ESFERA EMOCIONAL OU PSICOLÓGICA
Diminuição da libido, o desejo sexual
Diminuição da massa e da força muscular
Alterações do humor

\begin{tabular}{ccc}
\hline Diminuição da intensidade do orgasmo & $\begin{array}{c}\text { Diminuição de energia para atividades } \\
\text { anteriormente realizadas de forma natural }\end{array}$ & Irritabilidade \\
\hline Disfunção erétil (dificuldade de ereção) & Aumento da gordura corporal & Ansiedade \\
\hline $\begin{array}{c}\text { Diminuição do volume de esperma } \\
\text { ejaculado }\end{array}$ & Perda de pelos faciais e do corpo & Dificuldade de concentração da capacidade cognitiva \\
\hline- & Anemia & Perda de memória \\
\hline- & Letargia & Diminuição da orientação espacial \\
\hline- & Osteoporose & Alterações da sensação de vitalidade e \\
\hline- & Calorões & do bem-estar geral
\end{tabular}

Fonte: Autores com dados extraídos de Quadros e Schiavani (2005).

Vale salientar que, os itens acima listados, são os principais sintomas da andropausa, porém, é importante observar que nem sempre todas essas manifestações precisam estar presentes, ou seja, elas também podem surgir de forma isolada (Quadros \& Schiavani, 2005). O tratamento da andropausa requer uma avaliação minuciosa pelo médico urologista, uma vez que, é importante salientar que existem alguns efeitos adversos relacionados com a reposição de testosterona, a saber: ginecomastia, infertilidade, policitemia, apnéia obstrutiva do sono e patologia prostática (Glina, Puech-Leão, Reis \& Pagani, 2002). 
Góis, Santos e Araújo (2020) apontam que, dentro das representações sociais acerca da velhice masculina no que tange as mudanças no aspecto físico, além das perdas relacionadas aos âmbitos sexuais e reprodutivos, o indivíduo na fase da madurescência também tem reduzidas suas capacidades fisiológicas, como a perda de memória, força e equilíbrio, mobilidade reduzida, visão fragilizada e dores das mais diversas ordens, provenientes tanto de doenças, quanto da senescência propriamente dita.

A partir do exposto, subentende-se que durante o período caracterizado como meia idade, faz-se necessário analisar a interação que ocorre entre as mudanças físicas, biológicas e sociais, bem como suas respostas a essas alterações, com o intuito principal de entender como o homem as manifesta na fase da madurescência - ou meia-idade. Outrossim, compreender estas mudanças poderá auxiliar no entendimento das principais manifestações psicológicas decorrentes da fase.

\section{Aspectos Psicológicos}

O ciclo vital é próprio da composição orgânica e funcional do ser humano e, dessa forma, o processo de desenvolvimento permeia a vida desde o nascimento até a morte. Muitos teóricos dividem as fases desenvolvimentais em período de crescimento, maturidade e por fim o de declínio, sendo todas caracterizadas por perdas biológicas e sociais e, geralmente, marcadas pelas transições na pirâmide familiar. Diante disso, autores têm refutado a visão de que, diferentemente da infância e adolescência, a vida adulta seja um período de relativa estabilidade. (Gonçalves, Fagulha \& Ferreira, 2005; Sartori \& Zilberman, 2009; Virgolino et al., 2013).

Grande parte da literatura enfatiza que a meia-idade é, tanto para os homens como para as mulheres, um período de angústias normais do período, porém a maneira de vivenciá-las depende de fatores que são substancialmente pessoais e peculiares. Para Fraiman (2004), a idade adulta é o resultado dinâmico de um processo global de modificações incessantes, experimentadas em qualquer idade, que podem ser brandas ou abruptas, conscientes ou inconscientes, culturais, históricas, sociais, psicológicas ou biológicas. Quando conscientes, causam no indivíduo uma comparação, um diálogo e uma revisão, possibilitando uma nova probabilidade de organização de futuros confrontos (Fraiman, 2004).

Sartori e Zilberman (2009) descrevem essa fase como o início do período de declínio, frisando que, neste momento, ocorrem perdas não apenas biológicas, mas relacionadas a diversas questões no âmbito familiar, seja nas tarefas, no crescimento pessoal ou na função parental. Nesse sentido, as autoras referem que a depressão, a dependência e a desestruturação familiar são verificadas e, muitas vezes, esses sintomas estão associados a chamada síndrome do ninho vazio (SNV), decorrente do sofrimento gerado pela perda do papel da função parental com a saída dos filhos da casa dos pais (Sartori \& Zilberman, 2009).

Apesar da literatura demonstrar que o sexo feminino está mais vulnerável a apresentar sintomas da SNV, pois a mulher dedica muito do seu tempo aos filhos e, neste período da vida, perde a sua posição de cuidadora na base familiar, pesquisas têm apontado que a síndrome também acomete os homens (Tavares et al., 2004; Virgolino et al., 2013), muito embora, seja vivenciada distintamente. Transversalmente a isso, enquanto, muitas vezes, a mulher ressignifica a experiência, lançando-se a projetos os quais preteriu para atender os filhos, os homens tendem a um movimento contrário: eles optam por atividades domésticas e neles predomina um sentimento de acomodação; essa dissonância pode conduzir a conflitos entre os casais (Sartori \& Zilberman, 2009; Virgolino et al., 2013).

Sartori e Zilberman (2013) citam Hiedemann et al (1998) para apontar relações entre SNV e problemas conjugais; estes autores, em estudo longitudinal, analisaram riscos de separação ou divórcio em casais da meia-idade, concluindo que a SNV acarreta efeitos negativos na vida matrimonial. Não obstante, assinala-se que esses efeitos dependem da qualidade e da duração do relacionamento, nos bons casamentos a saída dos filhos de casa pode introduzir uma segunda lua-de- mel, já nos casamentos inseguros, em que casais resistem a união por causa das crianças, poderão não mais encontrar razões para 
prolongá-la (Papalia e Feldman,2006).

Ainda, Sartori e Zilberman (2009) afirmam que os homens sofrem muito na maturidade, porém não costumam externar seus sentimentos em função dos aspectos culturais relacionados à masculinidade. Esse comportamento resulta em aumento nos casos de alcoolismo, hospitalizações psiquiátricas, suicídios, além de sérios problemas de saúde física (Sartori \& Zilberman, 2009). As autoras mencionam ainda que as transformações neste período de desenvolvimento do homem são múltiplas e, por isso, não se pode ligar esses episódios à SNV. Justo e Calil (2006) aludem que o funcionamento hormonal, também, pode interferir nas manifestações depressivas.

Papalia e Feldman (2006) enunciam que embora Erikson, em seus conhecidos estudos sobre o desenvolvimento psicossocial, considerasse a formação de identidade como principal preocupação da adolescência, ela continua desenvolvendose, marcadamente, também, na meia-idade. Além disso, as autoras explicam, que a identidade pode constituir-se de múltiplos "selves possíveis", incluindo o self que a pessoa espera ser e o self que a pessoa tem medo de ser, ademais em fases de transição esses "selves" costumam ser revistos, podendo ser uma questão central na vida adulta (Papalia \& Feldman, 2006).

Destarte, a crise da meia-idade, assim como na adolescência, foi definida como uma crise de identidade. As autoras, citam Jacques e Levinson (1996) ao abordarem o conceito, pontuando que a consciência da mortalidade traz à tona a questão cronológica dos sonhos almejados e não realizados, ou que não trouxeram a satisfação esperada. Dessa forma, se quiserem mudar a direção, necessitam agir rapidamente para reestruturar suas vidas. (Papalia \& Feldman, 2006).

Entretanto, para que o estágio de desenvolvimento implique em uma crise ou não dependerá mais das circunstâncias individuais e dos recursos pessoais do que da idade, sendo que pessoas com ego resiliente vivenciam de maneira mais flexível e positiva eventos estressores (Papalia \& Feldman, 2006).

O conflito em renunciar sonhos, fantasias e aceitar o que foi conquistado até então, ou lançar-se a novas tentativas na vida profissional, amorosa ou familiar, atrelado ao sentimento de que "adiar será tarde demais", configuram a crise da meiaidade. Ainda, soma-se o enfrentamento da finitude do tempo e da vida ancorados por sentimento de frustração e patologias significativas determinando uma verdadeira crise pessoal, de natureza psicológica e existencial (Eizirik, Kapczinski \& Bassols, 2007)

Outro aspecto negativo que contribui para o desencadeamento da crise da meia idade é o medo do abandono e da desvalorização com a aproximação da velhice, muito porque a sociedade tende a desvalorizar e abandonar as pessoas que não produzem mais fisicamente, ao mesmo tempo, e por isso, a exigência pessoal do homem é potencializada. Isso pode contribuir para o desenvolvimento de doenças psicossomáticas relacionadas à negação da perda da identidade e desejo de retardar o envelhecer (Trindade, 2002).

Nesse sentido, Papalia \& Feldman (2006) trazem a teoria clássica de Erikson (1968), a cerca do funcionamento psicológico saudável e bem-sucedido na meia-idade correlaciona-se com a resolução da crise de geratividade versus estagnação, a qual geralmente advém dos contextos sociais e dos envolvimentos com os papéis assumidos. As autoras citam pesquisas as quais constataram que homens mais gerativos, profissional ou socialmente, são mais predispostos a formas maduras de enfrentamento, através, por exemplo, do altruísmo e humor, ao invés de utilizarem formas imaturas, como beber ou desenvolver hipocondria (Papalia \& Feldman, 2006).

Diante disso, observa-se que encarar o "declínio" da meia-idade - fruto de perdas biológicas, culturais e sociais - faz parte de um ciclo que exige ao homem adaptar-se a novas possibilidades existenciais. A meia-idade é um processo inevitável da natureza humana, o qual é atravessado por mudanças na forma de "se ver" e pelos papéis desempenhados na família e na sociedade. Conquanto, as transformações sejam definidas cultural e contextualmente, as reações são subjetivas, por isso, buscar compreender essa fase e seus significados permite vivenciá-la com uma maturidade estável. 


\section{Relação com o trabalho e aposentadoria}

$\mathrm{Na}$ literatura sobre o tema, percebe-se que o trabalho é um organizador psíquico e, geralmente, a meia-idade é um período do ápice do exercício do poder gerado por anos de trabalho. Assim, discorre-se que, muitas vezes, as gratificações narcísicas em relação as produções labutares transformam-se na principal fonte de gratificação compensadoras da realidade diária (Eizirik, Kapczinski \& Bassols, 2007).

Diante disso, o trabalho assume grande importância na vida do homem, especialmente nesta fase. Segundo o dicionário (Michaelis, 2020), a palavra trabalho origina-se do latim Tripaliare, que significa o ato de castigar com um utensílio de tortura chamado Tripalium. No seu significado original, o trabalho acarreta o sentindo de castigo e sacrifício, ou seja, está longe do que significa realização e prazer profissional como é muito falado. Na meia idade, depois de muitos anos de dedicação à empresa, os homens podem não se sentir tão satisfeitos e acabar percebendo que o próprio reconhecimento da empresa e dos outros não são mais suficientes para a realização profissional, também enquanto sujeitos (Mardegan Jr., 1992).

Atualmente, no contexto social em que vivemos, partimos do pressuposto de que os homens não devem ser sujeitos frágeis, o que os leva a sentirem-se mobilizados a não demonstrar afeições negativas quando se trata de sentimentos (Silva \& Silva, 2014). Segundo Eizirik, Kapczinski e Bassols (2007), na meia-idade, tanto executivos, quanto trabalhadores braçais, preferem a segurança e posição atingida às novas oportunidades, mesmo que melhores.

Albornoz (2000) e Peres (2014) descrevem o trabalho formal ou informal como sendo uma construção social que, através do passar dos anos, tomou espaço nas nossas emoções, realizações e satisfações voltadas à sociedade, fazendo-nos, desta forma, sentir-nos úteis. Assim, introduzido por um sistema de regras e leis decretadas ao longo da nossa sociedade, a aposentadoria é o marco inicial para considerar a velhice, significando o limite do corpo, do físico e/ou psíquico que não é mais possível responder a demanda de produção do capital. (Silva, 2008).

Madergan Jr. (1992) aponta que o modelo tradicional percorrido pela história se fixou no homem, tornando-o prisioneiro de si mesmo e programado para o dever, o trabalho e o auto sacrifício. Entretanto, é nessa fase vital em que o homem "idealizado" sente que todo seu esforço não foi suficiente para leva-lo a uma vida feliz e bem-sucedida, tendo, deste modo, que estar de acordo com o que jamais realizará.

No que tange ao trabalho, nesta fase da vida, percebe-se que ele pode ser o foco de uma crise, uma vez que para a sociedade contemporânea, é a esfera da vida mais vista por todos e que está constantemente sendo analisada; inclusive, pela própria pessoa que está passando pela crise. (Antunes \& Silva, 2013).

Ao enfrentar a passagem da meia-idade, o homem é chamado a refletir sobre seus sentimentos mais íntimos, ao mesmo tempo que, no seu local de trabalho, suas responsabilidades também acabam consequentemente aumentando. Para Mardegan Jr. (1992), o conflito é inevitável, pois sempre existiu a luta pelo alcance de poder e status, deixando de lado as emoções e sentimentos. Outro aspecto importante relacionado a esta fase, segundo o autor, é a questão do homem se sentir ameaçado pelos mais jovens, e também pelas máquinas dentro do seu próprio ambiente de trabalho.

Zanelli e Silva (2008) apresentam a possibilidade da aposentadoria ser observada sob dois pontos de vista. Um deles é o descanso remunerado, proporcionando o tempo livre para os interesses pessoais, como por exemplo, aproveitar com a família, viajar, praticar hobbies; enquanto de outro lado opera-se a redução da capacidade financeira, perda de status e de vínculos afetivos.

Assim, compreende-se que a transição do trabalho para a aposentadoria deve ser uma fase de readaptação, de um despertar incansável por novos interesses, suprindo a carência da rotina laboral por meio do maior convívio familiar, bem como, do desejo de descobertas por novas atividades que satisfaçam os sujeitos. 


\section{Conclusão}

À luz da literatura, a construção deste artigo tornou possível a investigação da madurescência masculina, tema que, apesar de possuir relevante importância, foi identificado em menor proporção de materiais recentes, se comparado à meiaidade feminina. Ante ao exposto, entendeu-se que esse período da vida do homem é repleto de transformações em todas as esferas - psicológicas, biológicas e sociais - e carece de atenção tanto quanto a mesma fase da vida da mulher.

Outrossim, observou-se que na chamada meia-idade o homem passa por uma série de mudanças que estão intrinsecamente ligadas à idade. Além das alterações biológicas como o aparecimento de rugas, queda de cabelo, e até mesmo da impotência sexual, há também consideráveis conflitos de ordem psicológica atrelados às mudanças do corpo, e não obstante, cobranças pessoais.

Evidenciou-se que os homens possuem mais resistência em demonstrar seus sentimentos e frustações e, por isso, é muito importante o diálogo e a transparência principalmente nas relações de conjugalidade, uma vez que, na meia idade, em grande parte das famílias, os filhos já saíram de casa, e a família acaba sendo reduzida, formada apenas pelo casal. O acolhimento, a atenção e compreensão do cônjuge são fundamentais nessa fase e os relacionamentos pessoais, familiares ou não, são essenciais para a saúde do indivíduo; a ausência dessas relações pode torná-lo incapaz de resolver uma infinidade de problemas e conflitos intrínsecos à fase.

Por fim, reitera-se a importância de estudos atuais sobre o tema e, fundamentalmente, de pesquisas que reforcem as principais diferenças da madurescência masculina e feminina, evidenciando todas as esferas abordadas.

\section{Referências}

Albornoz, S. (2000). O que é trabalho (6 $6^{\mathrm{a}}$ ed.). São Paulo, Brasil: Brasiliense.

Antunes, P. C., \& Silva, A. M. (2013). Elementos sobre a concepção da meia idade, no processo de envelhecimento humano. Revista Kairós Gerontologia, 16(5), 123-140. Disponível em: https://revistas.pucsp.br/index.php/kairos/article/viewFile/18926/14090

Bitencourt, B., Gallon, S., Batista, M., \& Piccinini, V. (2011). Para além do tempo de emprego: o sentido do trabalho no processo de aposentadoria. Revista de Ciências da Administração, 13(31), 30-57. DOI: 10.5007/2175-8077.2011v13n31p30

Bonaccorsi, A. C. (2001). Andropausa: insuficiência androgênica parcial do homem idoso. Uma revisão. Arquivos Brasileiros de Endocrinologia \& Metabologia, 45(2), 123-133. https://www.scielo.br/scielo.php?pid=S0004-27302001000200003\&script=sci_abstract\&tlng=pt

Brasil, Ministério da Saúde (2008). Política nacional de atenção integral à saúde do homem: Princípios e diretrizes. Secretaria de atenção a saúde. Departamento de ações programáticas estratégicas. http://bvsms.saude.gov.br/bvs/publicacoes/politica_nacional_atencao_saude_homem.pdf

Bulcao, C. B., Carange, E., Carvalho, H. P. de, Ferreira-França, J. B., Kligerman-Antunes, J. Backes, J., ... \& Sholl-Franco, A. (2004). Aspectos fisiológicos, cognitivos e psicossociais da senescência sexual. Ciências \& Cognição, v. 1, 54-75. http://pepsic.bvsalud.org/scielo.php?script=sci_arttext\&pid=S1806$58212004000100007 \& \operatorname{lng}=$ pt\&nrm=iso

Castro, T. P. de. (2016). Sobre Curso da Vida e Autoajuda: Contrastes entre a literatura de autoajuda brasileira, norte-americana e portuguesa. (Tese de Doutorado, Universidade Estadual de Campinas, Campinas). http://repositorio.unicamp.br/jspui/handle/REPOSIP/321655

Cordeiro, A. M., Oliveira, G. M. de., Rentería, J. M., Guimarães, C. A. (2007). Revisão sistemática: uma revisão narrativa. Revista do Colégio Brasileiro de Cirurgiões, 34(6), 428-431. DOI: 10.1590/S0100-69912007000600012

Correa, N. G., Alcaças, E. S. R., \& Assis, M. A. (2020). Linha do tempo para o envelhecimento: Análise de como a população masculina adulta se prepara para a senescência. Revista Científica UMC. Edição especial PIBIC. http://seer.umc.br/index.php/revistaumc/article/viewFile/1449/902

Eizirik, C., \& Bassols, A. M. S. (Orgs.) (2013). O Ciclo da Vida Humana: uma perspectiva psicodinâmica. (2a ed.). Porto Alegre, Brasil: Artmed.

Elias, C. S. R., Silva, L. A., Martins, M. T. S. L., Ramos, N. A. P. R., Souza, M. G. G., \& Hipólito, R. L. (2012) Quando chega o fim? Uma revisão narrative sobre terminalidade do período escolar para alunos deficientes mentais. SMAD: Revista Electrónica em Salud Mental, Alcohol y Drogas, (8)1, 48-53. DOI: 10.11606/issn.1806-6976.v8i1p48-53

Ferenhof, H. A. \& Fernandes, R. F. Desmistificando a revisão de literatura como base para redação científica: método SSF. (2016). Revista ACB: Biblioteconomia em Santa Catarina, Florianópolis, SC: v. 21, n. 3, p. 550-563. Disponível em: https://revista.acbsc.org.br/racb/article/view/1194

Ferreira, M. E. M. P. (2008). A meia idade e a alta modernidade. Construção Psicopedagógica, 16(13), 77-91. http://pepsic.bvsalud.org/scielo.php?script=sci_arttext\&pid=S1415-69542008000100005\&lng=pt\&tlng=pt. 
Filho, J. F. L., Baccaro, L. F. C., Fernandes, T., Conde, D. L., Costa-Paiva, L., \& Neto, A. M. P. (2015). Epidemiologia da menopausa e dos sintomas climatéricos em mulheres de uma região metropolitana no sudeste do Brasil: inquérito populacional domiciliar. Rev. Bras. Ginecol. Obstet. 37(4), 152-158. DOI: $10.1590 /$ SO100-720320150005282

Finkelstein, J. S., Lee, H., Burnett-Bowie, S. A. M., Carl Pallais, M. P. H. J., Yu, M. P. H. E., Borges, L. F., ... \& Leder, B. Z. (2013). Gonadal Steroids and Body Composition, Strength, and Sexual Function in Men. The New England Journal of Medicine, 369 (11), 1011-1022. DOI: 10.1056/NEJMc1313169.

Fraiman, A. (2004) Coisas da idade, Coleção Plenitude - Volume II- São Paulo, Alexa Cultural.

Freitas, M. C. de., Campos, T. D., \& Gil, C. A. (2017). Expectativas e concepções de trabalho na velhice em homens na meia-idade. Est. Inter. Psicol. 8(2), 4364. DOI: $10.5433 / 2236-6407.2016 v 8 n 2 p 43$.

Glina, S., Puech-Leão, P., Reis, J. M. S. M., \& Pagani, E. (2002). Disfunção sexual masculina: conceitos básicos: diagnóstico e tratamento. São Paulo, Brasil: Instituto H.Ellis.

Góis, E.C. P., Santos, J. V. O, \& Araújo, L. F. (2020). Representações sociais sobre a velhice masculina: Abordagens de homens idosos participantes de grupo de convivência. Revista Subjetividades, 20 (Especial 1): e9140. https://periodicos.unifor.br/rmes/article/view/e9140/pdf

Gomes, R., Nascimento, E. F. do., \& Araújo, F. C. de. (2007). Por que os homens buscam menos os serviços de saúde do que as mulheres? As explicações de homens com baixa escolaridade e homens com ensino superior. Cad. Saúde Pública, 23(3), 565-574. https://www.scielo.br/scielo.php?script=sci_arttext\&pid=S0102-311X2007000300015

Goncalves, B., Fagulha, T., \& Ferreira, A. (2005). A depressão nas mulheres de meia idade: estudo sobre as utentes dos cuidados de saúde primários. Psicologia, 19(1-2), 39-56. http://www.scielo.mec.pt/scielo.php?script=sci_abstract\&pid=S0874-20492005000100003\&lng=pt\&nrm=iso

IBGE. (2019). Portal do Instituto Brasileiro de Geografia e Estatística. https://agenciadenoticias.ibge.gov.br/agencia-sala-de-imprensa/2013-agencia-denoticias/releases/26104-em-2018-expectativa-de-vida-era-de-76-3-anos

Lemos, A. P., Ribeiro, C., Fernandes, J., Bernardes, K., \& Fernandes, R. (2017). Saúde do homem: os motivos da procura dos homens pelos serviços de saúde. Revista de Enfermagem, 11(Supl. 11), 4546-4553. DOI: 10.5205/reuol.11138-99362-1-SM.1111sup201714.

Justo, L. P., Calil, H. M. (2006). Depressão: o mesmo acometimento para homens e mulheres? Revista de Psiquiatria Clínica, 33(2), 74-79. DOI: $10.1590 /$ S0101-60832006000200007

Madergan Jr. E. (1992). A crise da Meia Idade no Homem: Um estudo exploratório dos Fatores Determinante. São Paulo, Brasil.

Michaelis. (2020). Dicionário brasileiro da língua portuguesa. (4ª ed.). São Paulo, Brasil: Melhoramentos.

Montero, G. J. (2015) Psicoanálisis de la madurescencia (Definición,metapsicología y clínica). The International Journal of Psychoanalysis. Buenos Aires, Argentina.

Motta, A. B. (2006). Visão antropológica do envelhecimento. In: Freitas. (Org.). Tratado de geriatria e gerontologia. (2 ${ }^{\mathrm{a}}$ ed.). Rio de Janeiro, Brasil: Guanabara.

Paiva, A. V. R. (2016). Personalidade e sintomatologia: meia-idade tardia e terceira-idade. Lisboa, Portugal.

Papalia, D., Olds, S., Feldman, R. (2006). Desenvolvimento Humano. 8a ed. São Paulo, Brasil: Artmed.

Peres, A. (2014). Empreendedorismo e envelhecimento: Perspectivas de uma nova relação de trabalho na maturidade. (Dissertação de Mestrado, Universidade São Judas Tadeu, São Paulo, Brasil). https://www.usjt.br/biblioteca/mono_disser/mono_diss/2016/362.pdf

Quadros, S., Schiavini, J. L. (2005). Urologia sem segredos. Rio de Janeiro, Brasil: SBU.

Rother, E. T. (2007). Revisão sistemática x revisão narrativa. Acta Paul. Enferm., 20(2), v-vi.https://www.scielo.br/scielo.php?pid=S0103$21002007000200001 \&$ script=sci_arttext

Sartori, A. C. R., Zilberman, M. L. (2009). Revisitando o conceito de síndrome do ninho vazio. Rev. Psiquiatr. Clin., 36(3), 112-121. https://www.scielo.br/scielo.php?script=sci_arttext\&pid=S0101-60832009000300005

Silva, L. P., Silva, C. (2014). Idade do Lobo. Revista Portal de Divulgação, 40(4), 49-58. http://webcache.googleusercontent.com/search?q=cache:WIbwtjx6bikJ:revistalongeviver.com.br/index.php/revistaportal/article/do wnload/444/444+\&cd=4\&hl $=\mathrm{pt}-\mathrm{BR} \& \mathrm{ct}=\mathrm{clnk} \& \mathrm{gl}=\mathrm{br}$

Silva L. R. F. (2008). Da velhice à terceira idade: o percurso histórico das identidades atreladas ao processo de envelhecimento. História, Ciências, SaúdeManguinhos, 15(1), 155-168. DOI: 10.1590/S0104-59702008000100009.

Tavares, M. B., Fuchs, F. C., Diligenti, F., Abreu, J. R. P. de., Rohde, L. A., Fuchs, S. C. (2004). Características de comportamento do filho único vs filho primogênito e não primogênito. Brazilian Journal of Psychiatry, 26(1), 17-23. DOI: 10.1590/S1516-44462004000100007

Thiago, C. C., Russo, J. A., Camargo Júnior, K. R. de. (2016). Hormônios, sexualidade e envelhecimento masculino: um estudo de imagens em websites. Comunicação, Saúde e Educação, 20(56), 37-50. DOI: 10.1590/1807-57622014.0031.

Trindade, E. (2002). Hermenêutica do existir do homem de meia-idade - paternidade, sexualidade e projetos de vida: um olhar à luz de Heidegger. (Tese de Doutorado, Faculdade de Filosofia, Ciências e Letras de Ribeirão Preto, Universidade de São Paulo, Ribeirão Preto). https://www.teses.usp.br/teses/disponiveis/59/59137/tde-09032003-105542/pt-br.php 
Research, Society and Development, v. 10, n. 5, e46210515271, 2021

(CC BY 4.0) | ISSN 2525-3409 | DOI: http://dx.doi.org/10.33448/rsd-v10i5.15271

Virgolino, F. S. S., Silva, A. B. da., Porto, S. C. A. S., Souza Neto, V. L. de., \& Saraiva, A. M. (2013). A mudança no ciclo familiar diante da síndrome do ninho vazio: uma revisão. Revista da Universidade Vale do Rio Verde, v. 11, 457-464. DOI: 10.5892/ruvrv.2013.111.457464.

Zanelli, J. C., Silva, N. (2008). Interação humana e gestão: a construção psicossocial das organizações de trabalho. São Pau lo, Brasil: Casa do Psicólogo 